\title{
Linx
}

Revue des linguistes de l'université Paris X Nanterre

$7 \mid 1995$

Saussure aujourd'hui

\section{La référence à Saussure chez G. Guillaume}

\section{André Dedet}

\section{OpenEdition}

Journals

Édition électronique

URL : http://journals.openedition.org/linx/1239

DOI : 10.4000/linx.1239

ISSN : 2118-9692

\section{Éditeur}

Presses universitaires de Paris Nanterre

\section{Édition imprimée}

Date de publication : 1 septembre 1995

Pagination : 461-467

ISSN : 0246-8743

\section{Référence électronique}

André Dedet, «La référence à Saussure chez G. Guillaume », Linx [En ligne], 7| 1995, mis en ligne le 24 juillet 2012, consulté le 10 décembre 2020. URL : http://journals.openedition.org/linx/1239; DOI https://doi.org/10.4000/linx.1239

Ce document a été généré automatiquement le 10 décembre 2020.

Département de Sciences du langage, Université Paris Ouest 


\title{
La référence à Saussure chez G. Guillaume
}

\author{
André Dedet
}

1 Un an après la publication posthume du Cours de linguistique générale ${ }^{1}$, Gustave Guillaume, en 1917, présentait, à l'Ecole pratique des Hautes Etudes, son mémoire sur Le problème de l'article ${ }^{2}$. Dans cet écrit, d'une grande qualité et particulièrement novateur, se trouvent déjà esquissées, sinon conceptualisées, la méthode et la représentation du langage qui seront développées dans les écrits suivants. C'est dire que la pensée de G. Guillaume s'est construite avant la parution des thèses saussuriennes, son mémoire étant terminé en 1916, année de parution du C.L.G. ${ }^{3}$. Cependant, il est à remarquer que G. Guillaume est un des premiers lecteurs de Saussure. Il le citera à deux reprises dans ce premier ouvrage important. Et ces deux citations nous semblent caractéristiques de la relation qu'il entretiendra, tout au long de sa réflexion linguistique, avec le saussurisme. Dès l'avantpropos, expliquant sa méthode comparative appliquée «à la partie formelle des langues » (p. 11), il renvoie de façon tout à fait générale à deux ouvrages. Tout d'abord à l' Introduction à l'étude comparative des langues indo-européennes ${ }^{4} \mathrm{~d}^{\text {Antoine Meillet, linguiste }}$ dont il s'est toujours considéré comme le fidèle disciple, et ensuite au C.L.G. qui venait de paraître, mais sa référence à Saussure, associée à une référence à Meillet, apparait bien plus comme un hommage que comme une référence méthodologique, tant elle réfère vaguement à la cinquième partie du Cours : « la linguistique rétrospective » qui traite des problèmes du prototype et de la reconstruction des langues (C.L.G. pp. 295-303). Immédiatement après cette référence, qui présente Saussure comme s'inscrivant dans la lignée de Meillet, G. Guillaume précise sa démarche :

«I1 n'est plus question de reconstruire l'original des langues différenciées, mais de discerner en vertu de quelles nécessités communes les systèmes que forment ces langues se sont créés et ont subsisté. Le principe de la méthode reste le même, mais il faut tenir compte de changements imposés par la différente nature de l'objet. Au lieu de correspondances entre phonèmes, il s'agit de correspondances entre systèmes »(p.12).

2 En termes saussuriens, on remarquera que G. Guillaume indique qu'il ne se place point sur l'axe des successivités sur lequel « on ne peut jamais considérer qu'une chose à la fois » 
(C.L.G., p.115), ni sur le plan de l'analyse diachronique qui étudie des «termes successifs » (id., p. 140) mais bien sur le plan de l'analyse statique qui étudie des états de langue. Et la deuxième référence dans cet ouvrage est là pour préciser cette option :

"C'est l'équilibre sans cesse renouvelé, entre la dominance et la résistance, qui permet au linguiste la considération statique, autrement dit l'attitude intellectuelle du synchroniste (v. Ferdinand de Saussure, Cours de linguistique générale, p. 117) «(p. 67).

Si G. Guillaume pour situer sa démarche réfère à la distinction saussurienne entre linguistique statique et linguistique évolutive, c'est pour immédiatement indiquer ce qui l'en différencie. Pour G. Guillaume l'état statique est un équilibre précaire résultant de tensions contraires, et toute l'attitude intellectuelle du synchroniste consistera à saisir ces forces en jeu, cette mécanique «transcendante » qui conduit à la transformation du système. C'est à la mise en évidence de ces mouvements de pensée constructeurs qu'il s'attachera et, pour autant que la langue soit conçue par Saussure comme un «mécanisme » fonctionnant par l'opposition des «entités délimitées » (C.L.G., p. 145), il ne s'intéressera aucunement à la description statique des relations entres les termes du système. Et c'est bien là, en dépit de la référence à Saussure, un point qui différenciera sa démarche de celle de la majorité des post-saussuriens. Guillaume considère que c'est par la comparaison d'états de langue successifs que l'on peut appréhender les mécanismes constructeurs du système et décrire la langue et non, comme le propose Saussure, par une mise en évidence des rapports syntagmatiques et paradigmatiques une fois les unités délimitées. Il soulignera à plusieurs reprises cette différence.

On peut ainsi lire ces premières références bien plus comme une marque reconnaissant la qualité des écrits saussuriens, en ce qu'ils ont beaucoup de similitudes avec ceux de Meillet, que comme une adhésion aux thèses spécifiques qu'ils développent.

Cette attitude de reconnaissance mais, en même temps, de distanciation se retrouvera quelques trente ans plus tard lorsqu'à plusieurs reprises Guillaume citera son illustre prédécesseur.

6 En 1944, quand le saussurisme sera devenu dominant, dans un article du Français Moderne, formulant cette idée, dès lors commune, que la langue est un système, G. Guillaume renverra au C.L.G., mais ici encore, de façon très significative comme lors de sa première référence, en associant Saussure et Meillet et en précisant que cette idée du Cours n'est qu'une reprise :

«L'idée que la langue est un système (...) on la rencontre énoncée avec beaucoup de force, dès les premières éditions, dans l'Introduction à l'étude de la grammaire comparative de Meillet, mais c'est dans le Cours de linguistique générale de Ferdinand de Saussure que la même idée devient l'un des piliers de la doctrine, généralement admise depuis... $»^{5}$.

7 Association à laquelle il tient assez pour qu'il la reprenne, à plusieurs reprises devant ses auditeurs, presque mot pour mot :

«L'idée déjà fortement exprimée par Meillet, notamment dans l'Introduction à l'étude des langues indo-européennes, «Chaque langue forme un système où tout se tient, et a un plan général d'une merveilleuse rigueur » (...), cette idée a connu une grande fortune à partir du jour où elle est devenue, après 1916, la pierre angulaire de la doctrine de Ferdinand de Saussure, telle qu'elle se dégage du Cours de linguistique générale rédigé par ses élèves ${ }^{6}$

8 Ne faudrait-il pas lire ici comme une amertume devant le succès du Cours qui a connu, en effet, une grande fortune principalement du fait qu'on a attribué à Saussure la paternité 
de l'idée que la langue est un système, succès qui relègue injustement Meillet et son fidèle disciple dans l'ombre? Une appréciation sur Saussure faite en 1945 conforte cette idée. Dans l'avant-propos à L'architectonique du temps dans les langues classiques Guillaume regrette que la linguistique ne se soit pas encore convertie en science théorique et reste, par bien des points, une science d'observation et ce, précise-t-il, «en dépit de l'enseignement de Ferdinand de Saussure, fort admiré et peu suivi ${ }^{7}$. Cette critique se manifeste clairement quand, en réponse à une de ces auditrices d'un séminaire de 1946, il remarque que «les savants de l'école genevoise " ne sont pas tous "très fidèles, dans l'esprit sinon dans la lettre, à l'enseignement de Ferdinand de Saussure $»^{8}$. Ces notations paraissent ici intéressantes parce qu'elles précisent ce que sera l'attitude constante de Guillaume par rapport au saussurisme, une attitude de respect pour les recherches du linguiste et une irritation devant les succès et le " tapage » des saussuriens qui méprisent ses travaux, ignorent ceux de Meillet et lisent mal ceux de Saussure lui-même! Irritation et impression, sans doute, d'une injustice très forte pour qu'en 1952 il reprenne, à peu près dans les mêmes termes qu'en 1944 et 46 ses remarques sur Saussure ! Guillaume écrit alors :

«Un large courant d'approbation a accueilli en 1916, quand parut le Cours de linguistique générale de Ferdinand de Saussure, l'opinion qu'y professe l'auteur que la langue est un système et doit être étudiée en elle-même comme telle »"

Pour ajouter quelques lignes plus loin :

«Bien avant que n'ait paru le livre de Saussure, Meillet avait écrit: "Chaque langue forme un système où tout se tient et a un plan d'une merveilleuse rigueur" $»^{10}$

Une telle insistance, une telle répétition presque mot à mot, quand bien même serait-elle courtoise, est bien le signe d'un dépit, celui résultant de la non-reconnaissance à leur juste valeur des travaux du maitre et sans doute de ceux de son disciple qui, il faut bien le reconnaître, restent confidentiels, de ce fidèle disciple qui pour soutenir sa démarche est obligé de rechercher la caution de celui dont les thèses sont en vogues. En 1946, il notera :

«Dans le Cours de linguistique générale, Ferdinand de Saussure déclare légitime le point de vue qu'avaient, d'une manière générale, adopté, avant la grammaire historique, les auteurs de grammaire didactique. La langue étant à tout moment un système, la grammaire doit avoir pour objet essentiel la description du système considéré ${ }^{11}$

Référence, ici encore, symptomatique de ce que la citation est là pour servir de caution mais aussi pour permettre un commentaire par lequel on fait remarquer que la démarche préconisée était déjà adoptée par les " auteurs de grammaire didactique » et que donc l'idée de système et d'état statique, qui fait alors le succès du C.L.G., ne peut être attribuée à Saussure. Sentiment d'une profonde injustice très compréhensible à cette époque où le structuralisme dominant érige en référence absolue le modèle linguistique d'analyse systématique attribué à Saussure, alors même que Guillaume est certainement l'un des premiers à avoir fondé sa recherche sur cette idée et sans doute à avoir produit le plus de résultats. C'est aussi ce qui semble transparaître dans les références à Saussure, par rapport auxquelles Guillaume précisera dans ses Conférences la position de la psychosystématique :

«Ferdinand de Saussure (...) a posé l'équation langage = langue + parole, laquelle portée à un plus haut degré de généralité devient: langage = psychisme + sémiologie $»^{12}$; "Saussure n'a pas fait état à un degré suffisant de la notion de discours et de l'opposition langue/discours » (ibid. p. 19).

Les critiques se précisent plus loin : 
« Dans notre terminologie - qui par là se distingue de la terminologie saussurienne en un point non dénué d'importance - langue et discours sont des termes recouvrant des réalités corrélatives » (ibid., p. 94).

G. Guillaume note alors que si pour le sujet parlant :

«le fait de langue a bénéficié de l'antécédence par rapports au fait de discours et cette antécédence est le grand fait de synchronie non signalé du reste, il faut le regretter, dans le Cours de linguistique générale ",

pour la formation historique, il en va inversement :

« et c'est de nouveau le grand fait, qui est de diachronie, - non signalé dans le Cours de linguistique générale - là le bénéfice de l'antécédence appartient au fait de discours » (id. p. 103).

11 C'est à partir de ces distinctions que Guillaume formulera les thèses de la psychosystématique, thèses qui s'opposent à l'analyse saussurienne et dépassent l'écueil du domaine syntaxique, écueil reconnu par Saussure lui-même lorsqu'il note :

«Mais il faut reconnaître que dans le domaine du syntagme il n'y a pas de limite tranchée entre le fait de langue, marque de l'usage collectif et le fait de parole, qui dépend de la liberté individuelle» (C.L.G., p. 173).

Pour Guillaume, qui distingue entre Langue et Discours et, conséquemment, entre unité de puissance et unité d'effet, c'est à l'unité de puissance qu'il convient de s'attacher car l'unité d'effet n'est qu'une unité de discours. C'est cette distinction qui déterminera son attitude par rapport à l'analyse syntaxique :

«L'unité d'effet se construit à partir des unités de puissance préconstruites et sa construction, ses possibilités de construction dépendent de ce que sont dans la langue, de par leur construction, les mots de puissance ».

Il découle de là que :

«toute étude du mécanisme constructif de phrase se subordonnera à toute considération préalable à la structure du mot $»^{13}$.

13 On évite ainsi l'écueil du syntaxique. Cette modification conceptuelle que promeut Guillaume est un dépassement du Saussurisme. Elle implique, non plus une distinction entre diachronie et synchronie mais que l'on ait une visée qui permette une étude de la « diachronie des synchronies ${ }^{14}$ que Guillaume rapproche de la notion de " panchronie » de Saussure ${ }^{15}$. Ce qui le conduit à remarquer que le C.L.G. "n'indique, ne préconise aucune technique appropriée à l'analyse du système $»^{16}$. Guillaume précise encore :

«A la simple description des systèmes recommandée par Saussure, on fait succéder

l'histoire des systèmes dont Saussure ne parle pas $»^{17}$.

Cette approche guillaumienne conduit à voir dans la référence à Saussure le signe d'une estime pour un prédécesseur dont on considère la démarche théorique comme importante mais surtout comme inachevée ${ }^{18}$. Cette référence dans chacune de ces occurrences est aussi comme un leitmotiv qui dit une irritation résultant de ce que cette immense statue élevée au linguiste genevois par des continuateurs peu inspirés ${ }^{19}$ projette une ombre qui occulte, tout à la fois, l'oeuvre de son maître Meillet et la sienne propre.

Pour terminer, il faut remarquer que ce qui paraît le plus important ce n'est peut-être pas la référence à Saussure dans l'oeuvre de Guillaume mais la non-référence. On peut en effet s'étonner à constater que Guillaume, en fin connaisseur de Saussure, ne fasse jamais état de cette affirmation plusieurs fois répétée dans le C.L.G., et qui pourtant conforte l'approche guillaumienne, à savoir que «tout est psychologique dans la langue $»^{20}$, approche psychologique que les successeurs de Saussure ne prendront pas souvent en compte, alors qu'elle sera le fondement de la recherche guillaumienne. 


\section{NOTES}

1. Ferdinand de Saussure, Cours de linguistique générale, Payot, Paris, 1916, (C.L.G.).

2. Gustave Guillaume, Le problème de l'article et sa solution dans la langue française, (1918), rééd., Librairie A-G. Nizet, Paris, Les Presses de 1 Université Laval, Québec, 1975.

3. Voir Roch Valin, in Introduction à Leçons (1948-1949, Série A), Les Presses de l'Université Laval, Québec, Librairie Klincksieck, Paris, 1971.

4. Antoine Meillet, Introduction à l'étude comparative des langues indo-européennes, Paris, Hachette, 4e édit., 1915.

5. Gustave Guillaume, Particularisation et généralisation dans le système des articles français, (Avr.-Mai 1944), repris in Langage et Science du langage, Les Presses de l'Université Laval, Québec, et Nizet, Paris, 1964 (L.S.L.).

6. Gustave Guillaume, Leçons, (1946-1947, Série C), Les Presses de 1 Université Laval, Québec / Presses universitaires de Lille, Lille, 1969, p. 57 ; voir aussi Leçons, (1945-1946, Série C) , Les presses de l'Université Laval / Presses universitaires de Lille, 1985, p. 6 (L6).

7. Gustave Guillaume, L'architectonique du temps dans les langues classiques, (1945), 2e édit., Librairie Champion, 1970. Guillaume, Leçons, 1945-1946, Série A, Presses universitaire de Lille / Presses de l'Université Laval, 1987, (L7), p. 65).

8. Gustave Guillaume, Leçons, 1945-1946, Série A, PUL / Presses de l'Université Laval, 1987, (L7), p. 67.

9. Gustave Guillaume, La langue est-elle ou n'est-elle Dans un système, Cahiers de linguistique structurale, NUl, 1952, Les Presses de l'Université Laval, reproduit in. L.S.L. pp. 220-240, p.

10. id. p. 222. Voir aussi Leçons, 1956-1957, Les Presses de l’Université Laval, Québec / Les Presses universitaires de Lille, Lille, 1982 (L5), p. 104.

11. Gustave Guillaume, Leçons (1946-1947, Série C), op. cit., p. 69. G. Guillaume semble renvoyer ici au Chap. II des «Principes généraux » où Saussure parlant des "grammairiens inspirés par les méthodes traditionnelles », parmi lesquels il inclut les grammairiens de Port-Royal, écrit : « Il est curieux de constater que leur point de vue, sur la question qui nous occupe, est absolument irréprochable », (p.118). En 1949, Guillaume précise sa pensée en référant au C.L.G. grâce auquel, dit-il « on a découvert que l'état de structure d'une langue procède d'un jeu de causes bien plus compliqué et bien plus savant que celui que décrit d'ordinaire, et à peu près exclusivement, la grammaire historique ", Leçons (1949-1950, Série A), Les Presses de l'Université Laval, Québec / Librairie Klincksieck, Paris, 1974 (L4), p. 14.

12. Gustave Guillaume, Psycho-systématique du langage, (1948-1949, Série B), Les Presses de l'Université Laval, Québec 1971. Voir aussi L4, pp. 68-69.

13. Gustave Guillaume, Psycho-systématique du langage, op. cit., p. 30.

14. Gustave Guillaume, L6, p. 20. (7 déc. 1945).

15. Ferdinand de Saussure, C.L.G., pp. 134-135. I1 s'agit bien pour G. Guillaume de considérer la grammaire générale comme un ensemble de « principes généraux existants indépendamment des faits concrets » (C.L.G., p. 135).

16. Gustave Guillaume, L3, p. 224 (3 juin 1949).

17. Gustave Guillaume, L6, p. 49, (4 janv. 1946). 
18. a) Le C.L.G. «a été si fécond qu'il n'a pas fini d'exercer son influence " (L3, 19 nov. 1948), „C'est un livre de chevet » (L7, p. 6, 15 nov. 1945 et L6, p. 11, 23 nov. 1945), « il invite à la méditation profonde » (L7, p. 11) ;

b) « Une langue est un système et ne peut être autre chose qu'un système. Pour bien le concevoir, il est nécessaire d'introduire des notions, d'une importance capitale, dont le «Cours de linguistique générale » ne fait pas état », L4, (24 nov. 1949), p. 16.

19. «La doctrine de Ferdinand de Saussure (...) a eu un vif succès mais n'a trouvé dans l'application que peu de serviteurs » (L6, p. 4, 16 nov. 1945); cf. note 7. En 1952, Guillaume écrit : «Lecteur dès son apparition du «Cours de linguistique générale » de Saussure, il nous a semblé, dès ce moment et toujours depuis, que la tâche de la preuve et de l'explication complète en la matière était un legs du maître à ses disciples ", L.S.L., p. 221.

20. Ferdinand de Saussure, C.L.G., p. 21, voir aussi p. 32 et p. 37. 